\title{
The Node Reliability Approach to Broadcasting in Manets: Raising Reliability With Low End-to-End Delay
}

\author{
Talmai Oliveira, Fabíola Greve* \\ talmai@ufba.br, fabiola@ufba.br \\ Computer Science Department (DCC), \\ Mechatronics Graduate Program, \\ Federal University of Bahia, \\ Salvador BA 40.170-110, BRAZIL
}

\begin{abstract}
This paper evaluates how well a significant number of broadcasting protocols for MANETs behave when under a realistic scenario of momentary failures and topology changes, which is represented by an omission fault model. Reliable protocols - which use additional mechanisms to ensure higher delivery rates beyond besteffort guarantees - have been proposed, but they exhibit unacceptably high end-to-end delays. As a result of the study conducted, a new mechanism that helps to enhance the reliability of deterministic broadcasting protocols is proposed. The mechanism allows for scalability, and is capable of ensuring good delivery rates (in spite of failures) while maintaining lower end-to-end delays. Simulation results demonstrate the efficacy of the mechanism.
\end{abstract}

\section{Introduction}

A mobile ad hoc network (MANET) consists of a group of mobile hosts (also called nodes) that are capable of communication restricted to their wireless transmission range. The lack of fixed and wired gateways (base stations) forces cooperation between the nodes every time a message has to be forwarded. But, whenever messages overlap, collisions may occur, preventing correct reception. Many other factors also impede correct message transmission and reception as well, including hardware failure, battery exhaustion and node mobility [2]. Thus, ensuring reliable

\footnotetext{
${ }^{*}$ Supported by grants from CNPQ - Brazil and FAPESB Bahia/Brazil.

$1-4244-1455-5 / 07 / \$ 25.00$ C 2007 IEEE
}

communication between nodes is a major challenge in a MANET.

Broadcasting refers to the process by which one node sends messages to all other nodes in the network. It is an essential operation, since it may be used to collect global information, to support addressing algorithms and to help routing protocols [13]. In spite of the importance of the broadcasting primitive, not so much attention has been devoted to reliability requirements beyond best-effort in MANETs [3]. A large number of protocols for broadcasting in MANETs exist and previous studies reveals that most of them are very mobile resilient and support well congestion and collisions [5]. Although these factors can be considered as faults, they are not sufficient to denote all the possible fault scenarios that affect MANETs, such as link failures, network partitions, topology change during broadcasts and momentary node failures. Indeed, most of these protocols for MANETs consider failstop failures, but in real systems this is not always the case. In our opinion, it is the omission failure model where nodes fails by crashing (prematurely halting) or by sending/receiving only a subset of the messages that it actually attempts to send/receive - that appropriately represents real fault scenarios.

This paper evaluates how well a significant number of broadcasting protocols for MANETs behave when placed in an omission fail-prone scenario. The studies consist of measuring the reliability, the forwarding ratio and the end-to-end delay of the protocols when $0 \%, 25 \%$ and $50 \%$ of the nodes fail. The protocols in study were separated based on the use of additional mechanisms to attempt to ensure higher delivery rates beyond best-effort. The protocols that had these mechanisms (defined as reliable protocols) 
were Double-Covered Broadcast [9], EraMobile [10] and Reliable Broadcast [1]. The ones that did not (defined as non-reliable protocols) were $\mathrm{Wu}$ and Li's protocol's [15], Scalable Broadcasting Algorithm [11] and Dominant Pruning [7]. The evaluation experiments conducted show that even in an omission fail-prone scenario, the reliable protocols can achieve good delivery rates, but at the expense of a significant endto-end delay. Non-reliable protocols, for their turn, halt rapidly but were highly impacted by node failures and were not capable of maintaining high delivery rates.

As a result of the studies, the paper presents a new mechanism, the RELIABILITY AND STABILITY VERIFICATION PROTOCOL, namely R.S.V.P., capable of ensuring good delivery rates based only on the local perception that the node has on the network and not on global information nor on a specific model of nodes behavior; thus it allows for scalability. Moreover, protocols enhanced by R.S.V.P. tolerate better omission faults. Simulation results indicate that all enhanced protocols had an expressive delivery ratio increase and much lower end-to-end delays (when compared against the results of reliable broadcasting protocols).

The rest of the paper is organized as follows. Section 2 describes the system model and evaluation scenarios. Section 3 presents the reliable protocols and their performance results. Section 4 presents R.S.V.P.. Section 5 describes the non-reliable protocols and their performance results, with and without R.S.V.P. Section 6 compares the performance of R.S.V.P.enabled protocols against that of the reliable protocols. Finally, Section 7 concludes the paper.

\section{System Model and Evaluation Scenario}

Manet Model. A MANET is a graph $G=(V, E)$ in which $V$ represents a set of mobile nodes and $E$ represents a set of edges. An edge $(x, y)$ is defined whenever two nodes $x$ and $y$ are within their wireless transmission range; so that, $x$ and $y$ are considered neighbors. Simulations were executed by the NS-2 network simulator (version 2.30), where nodes were confined within $1300 \times 1300 \mathrm{~m}^{2}$ and had a constant transmission range of $250 \mathrm{~m}$. For the radio propagation model we used the Two-Ray Ground Reflection model, while the MAC layer followed the IEEE 802.11 specification with no RTS/CTS/ACK for all message transmissions. The movement pattern of each node follows the Gauss-Markov mobility model with maximum of 18 second pause times. We used BonnMotion v1.3a as our mobility scenario generator. To allow for proper initialization and settling, we allow 3000 seconds of node movement without any kind of message exchange. Each simulation then ran for a total of 500 seconds. In the first 100 seconds only "Hello" type messages are sent to allow for updated local topology information to be exchanged throughout the network. It is during the next 100 seconds that we configure nodes to broadcast data messages as well. For the last 300 seconds no new data messages are broadcasted, but nodes still exchange "Hello" messages, retransmit buffered messages as needed and move. This is to allow for proper message delivery termination, such as unsent queued messages, as well as possible re-transmission attempts. Each simulation was repeated 20 times to achieve the $95 \%$ confidence interval for the results. Values used for the simulation such as broadcast rate $(10 \mathrm{msg} / \mathrm{s})$ and node speed $(1 \mathrm{~m} / \mathrm{s})$ were determined based on results obtained through previous simulations that had the best overall effect on every metric measured and permitted the most stable and reliable communication.

Fault Model. Most deterministic broadcasting protocols are resilient to fail-stop failures due to the fact that these protocols use constant neighborhood set exchange between nodes. Thus, a faulty node can only interfere for a short time during the broadcasting process. Shortly after the failure, all neighboring nodes will detect the fault and in future broadcasts, the node (which has now crashed), will no longer be involved. Using a fail-stop failure model is, in our opinion, inadequate to analyze faults when simulating broadcasting protocols. Thus, unlike any other work we have seen before, we have implemented an omission fault model in order to simulate a real world scenario characterized by interference introduced by the environment, link instability and transmission failure due to node movement. This model is also applicable to probabilistic broadcasting protocols. In our implementation, a faulty node will fail to send messages with large payloads, such as broadcast messages, but will still be able to exchange neighborhood and control messages. This implements an omission fault model and also helps to stress those protocols that assume a correct behavior on the reception and transmission during a broadcast, specially by some special set of nodes such as the gateway nodes. On the other hand, this fault model will favor those protocols that use additional mechanisms to properly identify message reception by neighboring nodes. So, during each of the runs, an uniformly randomly selected set of nodes will fail by omission. The exact number of nodes that fail on any given run depends on the percentage of failed nodes which can be $0 \%$ (failure free), $25 \%$ (a realistic scenario of faults) and 50\% (a worst case scenario, able to stress the limits of the protocols). Although 
high, the $50 \%$ failure scenario is important as it has been proved that to solve distributed problems with stronger reliability requirements, like consensus and atomic broadcast, this is the highest number of nodes that can fail [12]. It is important to note that, in parallel with the omission faults simulated by our model, other failures still keep occurring during the execution. For example, transmitted messages frequently are dropped since, after all, the radio propagation model used (in NS-2) allows for transmission errors and nodes are mobile. In order to correctly compare the protocols, all were simulated under the same set of mobility and fault patterns, including the exact same broadcast message sending times.

The Metrics. We have defined three metrics with which we have divided the evaluation studies. The metrics are reliability, forwarding ratio and end-to-end delay. Since our main priority is analyzing the reliability of the protocols, both energy concerns and protocol overheadrelated metrics (such as "hello" message exchange) were not taken into consideration.

- Reliability: A high delivery ratio is the primary goal of any broadcast protocol. It will demonstrate not only if the broadcast protocol in question does what it is supposed to do, but will help to show how each protocol deals with failure.

- Forwarding Ratio: Protocol efficiency is given by the number of gateway nodes that re-transmit and take an active role in the broadcast. Therefore, an efficient broadcast protocol is one that uses the lowest number of gateways to reach the highest number of nodes. But with the induced failure of nodes, efficiency is better measured as a ratio of the number of nodes that received a packet to the number of nodes that acted as gateways. We denominate this the forwarding ratio.

- End-to-End Delay: This metric normally is used in conjunction with the others to help understand how congestion has affected the protocols, since it measures how long it takes any given packet to reach every node.

\section{Performance of Reliable Protocols}

This section describes each of the chosen reliable broadcasting protocols and then presents their performance results when under different failure scenarios.

Double-Covered Broadcast (DCB): Lou and Wu's goal when proposing the double-covered broadcasting protocol (namely, DCB) [9] was to reduce the number of forwarding nodes without sacrificing the broadcast delivery ratio. It is classified as a neighborhood designating protocol since it piggybacks selected gateways nodes in broadcast messages. By selecting a set of gateway nodes where not only every 2hop node is covered, but also where all 1-hop nodes are covered by at least 2 forwarding neighbors, it benefits from the broadcast redundancy to improve reliability. Additionally, in DCB the re-transmission of the message by the gateways node serves as an ACK of correct message reception to the original sending node. If sender fails to detect/overhear all of the retransmissions, it assumes that a transmission failure occurred. The sender will keep re-sending the message until all forward nodes have re-transmitted or until a threshold is reached. By double-covering, DCB assumes that at least two transmissions will reach the nodes, therefore this redundancy prevents a single transmission error from interfering on message transmission and reception.

EraMobile: EraMobile [10] is presented as a deterministic alternative to the probabilistic gossipbased broadcasting. The basic idea behind gossipbased broadcasting is to have each node periodically exchange knowledge of received messages with a random set of other nodes. Gossip-based protocols are then very resilient to node mobility and failure. The authors of EraMobile exploit the broadcast nature of wireless communication and periodically exchange gossip messages with all 1-hop neighbors. Whenever a node detects that it has not received an specific message, it will request it through a broadcast request. EraMobile is also equipped with other performance enhancing mechanisms such as random jitters on gossip sending, traffic reduction based on local node density levels, and even an adaptation mechanism for energy conservation whenever local traffic is idle.

Reliable Broadcasting (RB): Proposed in 1995, the authors of the Reliable Broadcasting Protocol (namely, RB) [1] based their work on flooding. Whenever a node transmits a message, each receiver should return an acknowledgement to the sender (if it is not a duplicate message), and has now the responsibility to forward the message. If the sender does not receive an ACK it retransmits the message. Central to the protocol is the maintenance of a history of messages received. When nodes detect new neighbors, it uses a handshake procedure to exchange histories. On finding a missing message, a node can ask the other to supply it.

\subsection{Simulation Results [Reliable]}

In Figure 1 we can see the results of the three protocols. The first column regards the Double-Covered protocol, the second EraMobile and the third Reliable Broadcasting. We can see that regarding delivery ratio, $\mathrm{RB}$ is the protocol with the highest coverage reaching a minimum of $94.5 \%$ of the nodes in the worst case 
(a) Double-Covered
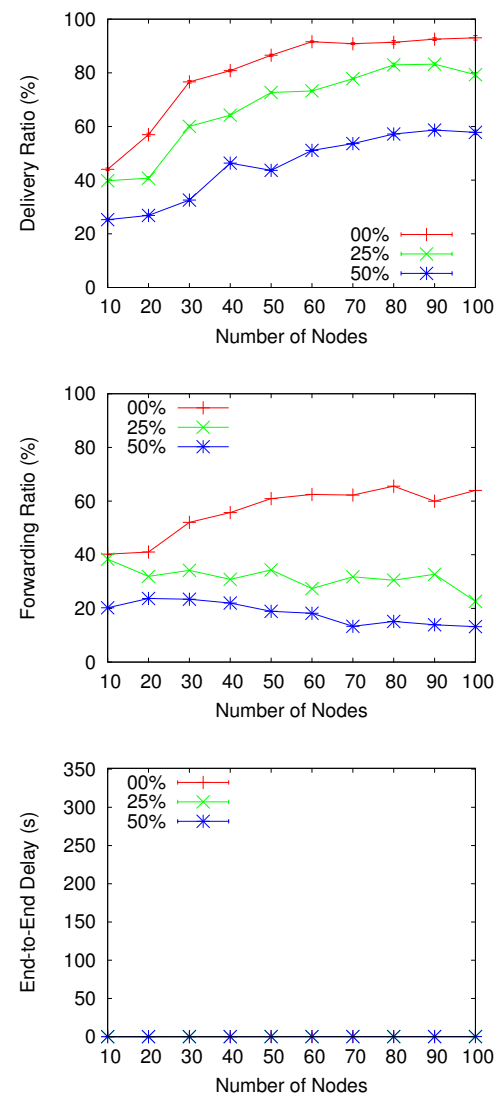

(b) EraMobile
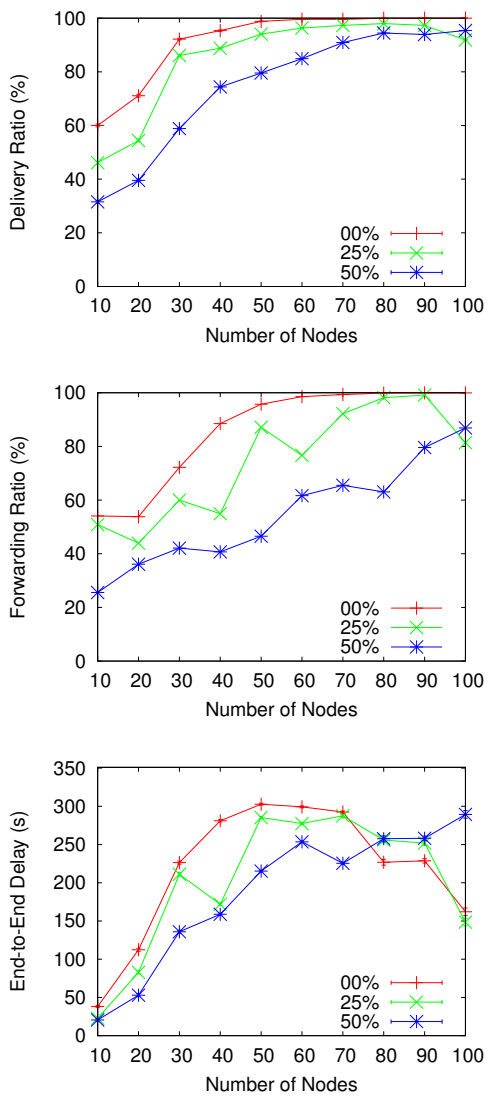

(c) Reliable Broadcasting
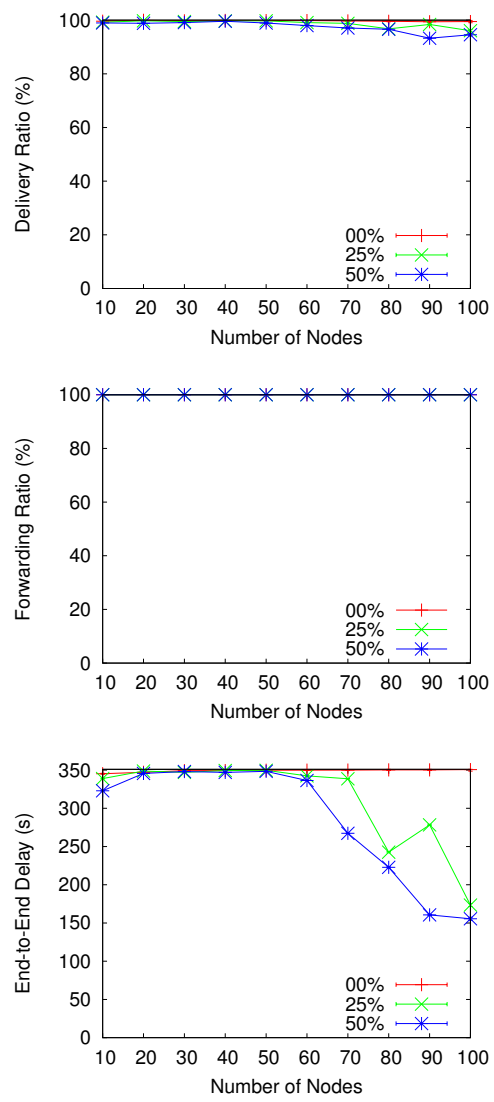

Figure 1. Effects of Node Failure on Reliable Protocols

scenario (100 nodes, $50 \%$ failure), and $100 \%$ of the nodes in all other scenarios. While EraMobile is capable of maintaining similar rates, it does not handle failures as well as RB. Nevertheless, with 40 or more nodes at least $75 \%$ of the network received, regardless of failures. Of all reliable protocols, DCB is the one with the worst delivery ratios. It is also the one most affected by failures. It is only capable of reaching the $85 \%+$ rates when there are no failures and at least 50 nodes, but in any fail-induced scenario this never happens. With $50 \%$ of the nodes failed, message delivery ratio reaches at most $60 \%$ of the network. The forwarding ratio results helps understand the overall cost to achieve the delivery ratios. While DCB involves less than $70 \%$ of the receiving nodes in the forwarding process, both EraMobile and RB involve at times all of the nodes. Finally, when we analyze the end-toend delay of the protocols we can clearly notice the impact that the retransmissions attempts have on the latency. While DCB has delay less than $0.2 s$, RB reaches $351 s$ and EraMobile 300s. We present in the next section a simple mechanism that, when used by existing non-reliable broadcasting protocols, can raise reliability while maintaining low end-to-end delay.

\section{Reliability and Stability Verification Protocol (R.S.V.P)}

Principle. R.S.V.P was thought up as a mechanism capable of augmenting reliability in deterministic broadcasting protocols. The main idea behind it is to re-transmit a message until all 2-hop nodes confirm reception. During this re-transmission process it also rates neighboring nodes according to the number of times that a message has to be re-transmitted in order to reach all 1-hop and 2-hop nodes. From a nodes' point of view it is of the utmost importance that surrounding 1-hop nodes be the ones that receive most attention. This is due to the fact that those nodes are the ones who are to forward any future broadcasts. But, in order to determine if these 1-hop nodes are actually forwarding the messages, message reception 
acknowledgements will have to be sent by the 2-hop neighbors. These acknowledgements can be piggybacked to reduce message transmission. A node can obtain its neighborhood information by periodically sending and exchanging "hello" messages. By sending "hello" messages containing not only the node identification but also its list of neighbors, receiving nodes can learn the topology information within 2 hops. By also piggy-backing message acknowledgements, nodes will be able to confirm message reception and define node reliability values, as well as maintain an updated perception of neighboring nodes. Each node maintains a perceived reliability vector $P R$ which will contain all nodes in its 1-hop vicinity. Thus $P R_{i}(v)$ defines the perceived reliability that node $i$ has of node $v$. At any moment, any given node may compare the perceived reliability values of his neighbors, can establish nodes that he trusts more than other or even determine a minimum reliability threshold by which nodes with lower $P R$ values are ignored. Every node also maintains a boolean vector of $n$ bits ( $n$ equal to the total number of 1-hop and 2-hop neighbors) for every message sent, that indicates whether the other $n$ nodes have received that message. This vector is called the confirmed reception vector $C R$ and is updated every time a "hello" message arrives containing a message confirmation acknowledgment. Thus, $C R_{i}^{m}(v)$ refers to the vector of node $i$ concerning reception of message $m$ by node $v$. If a bit of the vector is set it means that the corresponding node has confirmed reception of the message. Since a node cannot un-confirm reception, once set it guarantees reception.

R.S.V.P requires that at every "hello" transmission, node $k$ piggy-back $C R_{k}^{m}$. When a node $j$ receives the vector $C R_{k}^{m}$ for the first time, it sets its own $C R_{j}^{m}$ equal to the received knowledge vector and sets its own bit to express the reception (in other words, $C R_{j}^{m}(j)=$ true). Next time node $j$ transmits a "hello" message, it will include its updated copy of $C R_{j}^{m}$. By piggy-backing a node's confirmed reception vector, receiving nodes are able to detect which 2-hop nodes have not yet received that message. A node will keep re-transmitting $m$ until all 2-hop neighbors confirm reception. When this condition is finally verified, the message can be inhibited of future re-transmissions by removal from internal buffers. A maximum number of retries can also be used to properly terminate the broadcasting process.

Detailed Description. R.S.V.P will now be described as can be seen in Algorithm 1. The process by which neighborhood information is updated is specific to the broadcasting protocol used and therefore will not be defined in the following algorithm, but we assume that this information is available to our mechanism in order to determine neighbors' status.

Constant Symbols. The following constants will be used by the mechanism.

- BCAST: a generic deterministic broadcasting protocol responsible for determining a set of forwarding nodes;

- initial_perceived_reliability: value of the initial perceived reliability;

- decay_value: amount by which the perceived reliability value decays with time;

- increase_value: amount by which the perceived reliability value is incremented every time a new "hello" message is received;

- decrease_value: penalty amount value by which the perceived reliability value is decreased when nodes do not confirm reception;

- $\tau$ : internal timeout between consecutive neighborhood update procedure execution ("hello" transmission).

Variable Symbols. The following variables are setup and used by the mechanism to define reliability.

- $N(v)$ : set of 1-hop neighbors of node $v$;

- $F_{v}$ : set of forwarding nodes from nodes' $v$ point of view calculated using $B C A S T$;

- $P R_{v}(u)$ : the perceived reliability of node $u$ from nodes' $v$ point of view;

- $C R_{v}^{m}(u)$ : boolean reception vector which indicates reception of message $m$ sent by node $v$ to node $u$;

- $X_{v}^{m}$ : set of nodes which have not yet confirmed reception of a message $m$ sent by $v$.

When initialized, all perceived reliability values are set to initial_perceived_reliability (lines 2-4). Every time a new neighbor is added to the neighborhood set its value is also initialized. The value set of initial_perceived_reliability has to be well chosen as well. The experiments conducted by [6] showed that very recent neighbors not necessarily meant a working communication link. That is why the lower the initial value, the more we favor older neighbors over new ones. For every message $m$ sent for the first time, the confirmed reception vector $C R_{v}^{m}$ is initialized as well by setting all values to false to indicate that no neighbor has yet received that message, in the same manner, the set $X_{v}^{m}$ is set to all 1-hop and 2-hop neighbors (lines 7-10). Since every "hello" message has a confirmed reception vector piggybacked, this information is analyzed in order to update the status of the neighboring nodes that have not yet confirmed reception (line 14). At this moment the perceived reliability value is also updated to reflect the fact that the neighbor is still up, by incrementing it with increase_value (line 13). Since a typical deterministic broadcasting protocol needs to update its neighborhood view periodically, we will assume that this occurs after every timeout $\tau$. It 


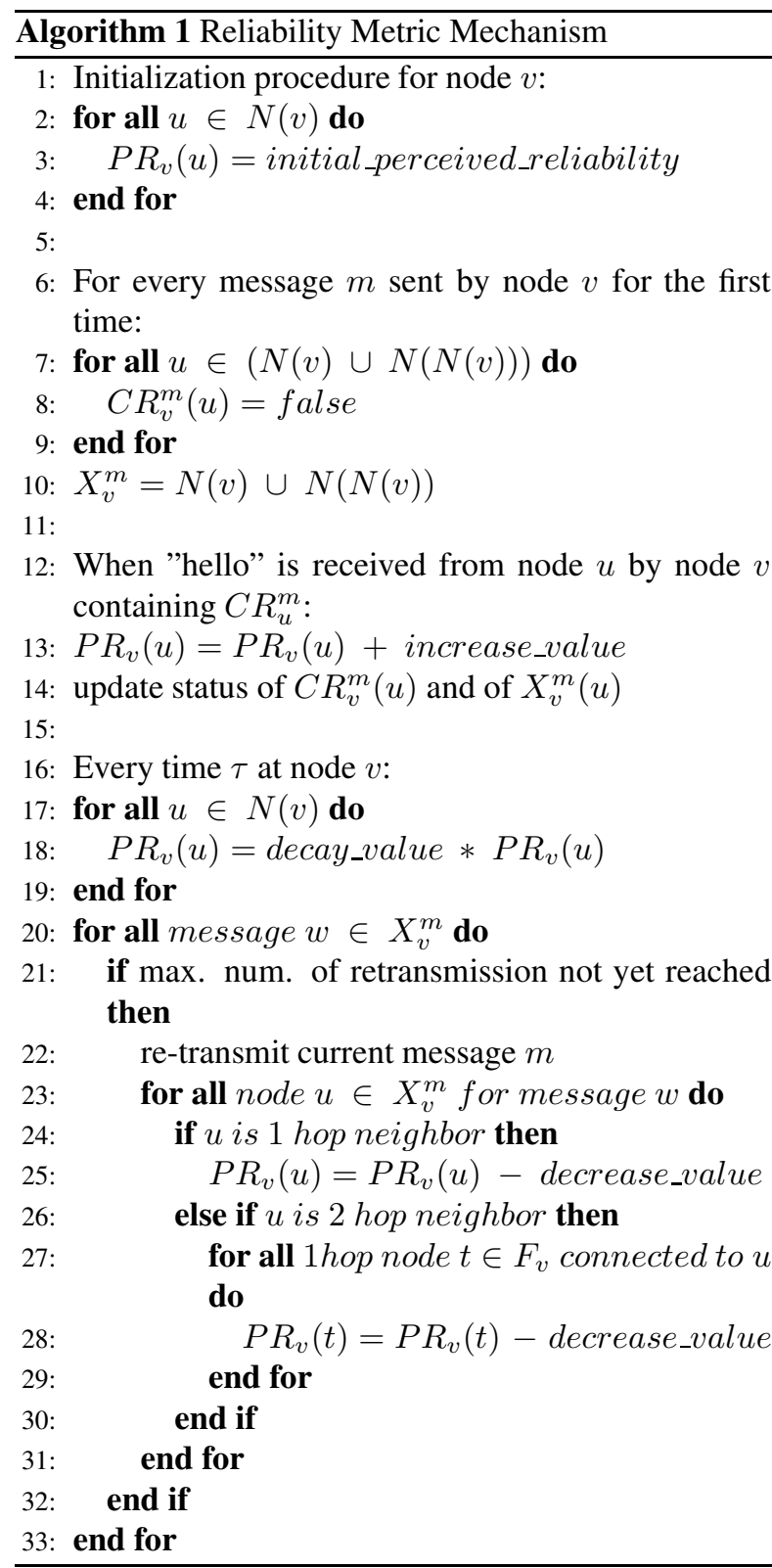

is also at this moment that the perceived reliability value is updated. Initially to reflect the fact that the perceived reliability should decay with time, we apply a decay_value (lines 17-19). Every time a neighbor does not confirm reception, a sender node can conclude that either it is his fault or it is the receivers fault. After all, ensuring effective communication between nodes is a major challenge regarding wireless communication. Thus the perceived reliability value must be updated. If it is a 1-hop neighbor $u$ that does not confirm reception of a message sent by $v, P R_{v}(u)$ will decrease by decrease_value (line 25). If it is a 2 -hop neighbor, then every 1-hop neighbor that is directly connected to the 2-hop node and belongs to the set of forwarding nodes will be penalized by decreasing the perceived reliability of that node by decrease_value (line 28). This is yet another point of the algorithm that depends on the $B C A S T$ protocol, this time for the selection of gateway nodes. This is necessary since our mechanism needs to establish which 1-hop neighbors are reliable, and if this gateway neighbor seems unable to correctly forward a message, it must be penalized.

\section{Performance of Non-Reliable Protocols}

In order to evaluate the performance and behavior of R.S.V.P, we simulated and compared its use in three well known deterministic broadcasting protocols: $\mathrm{Wu}$ and Li's protocol's [15], Scalable Broadcasting Algorithm [11] and Dominant Pruning [7]. In all those protocols R.S.V.P can be used by incorporating the supplied reliability values into the forwarding node set selection process that already exist in each individual algorithm. Therefore, if the reliability value of a node is below some pre-determined threshold, that node is removed from the possible set of gateway nodes and the selection procedure is restarted.

Wu and $\mathrm{Li}: \mathrm{Wu}$ and $\mathrm{Li}$ [15] proposed a deterministic self-pruning algorithm that has a simple selection process and relies on constant neighborhood set exchange between nodes: a node is marked as a gateway if it has two neighbors that are not directly connected. $\mathrm{Wu}$ and Li's protocol is well known and has been used and extended by many others [4, 5, 14]. But these works where all inspired on reducing the number of gateways nodes and on increasing broadcasting efficiency; not necessarily on achieving high message delivery ratio. Simulation results clearly show that although older, the original protocol still ensures higher message coverage [5]. This is why we chose it over the newer protocols.

In Figure 2 we can note the differences between $\mathrm{Wu}$ and Li's protocol running with and without the usage of R.S.V.P. While the R.S.V.P-enabled version had delivery rates up to $100 \%$ percent, without it they barely reached $60 \%$. On average, the extended version when on a network with at least 50 nodes was able to deliver messages to $79 \%-91 \%$ of the nodes. But using R.S.V.P also has its downside. The forwarding ratio which was always below the $50 \%$ mark, jumped to at least $90 \%$, involving up to $100 \%$ of the receiving nodes. The endto-end delay also increase quite a bit, especially when in a sparse network, but lowered to the $8-15$ second range starting from 60 nodes.

Scalable Broadcast Algorithm (SBA): The main idea of the deterministic self-pruning broadcasting algorithm 
(a) $0 \%$
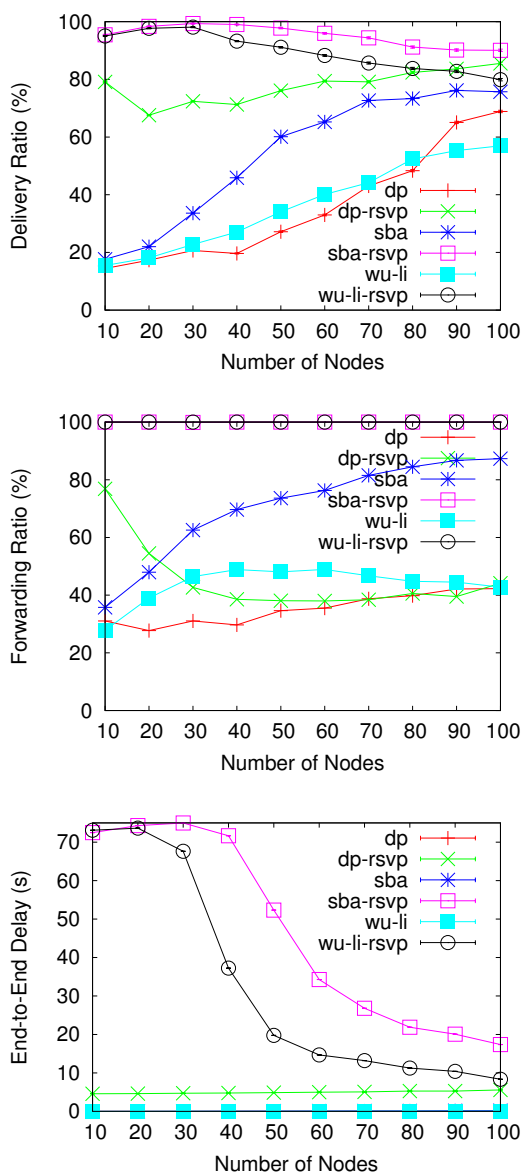

(b) $25 \%$
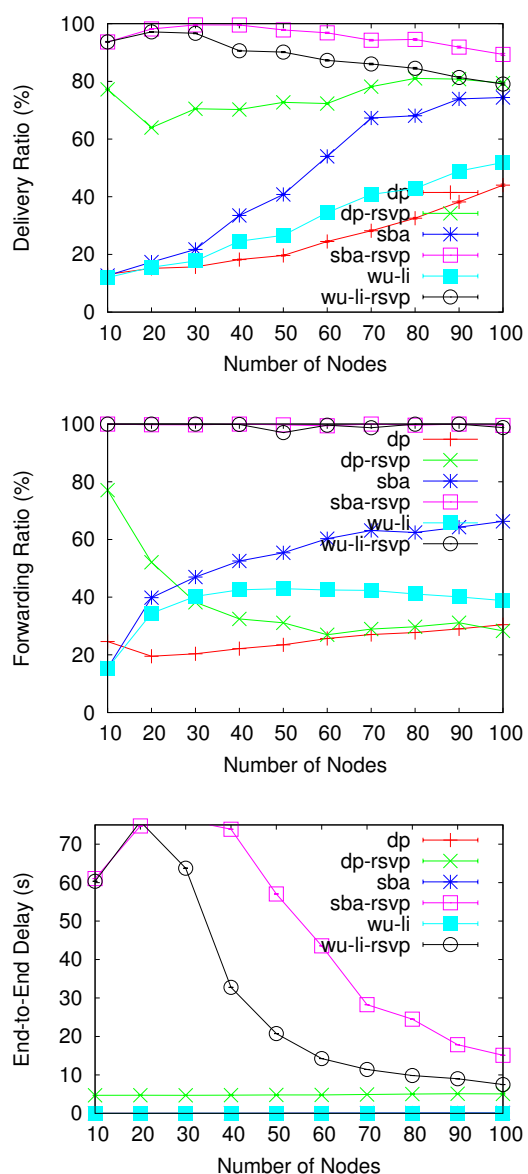

(c) $50 \%$
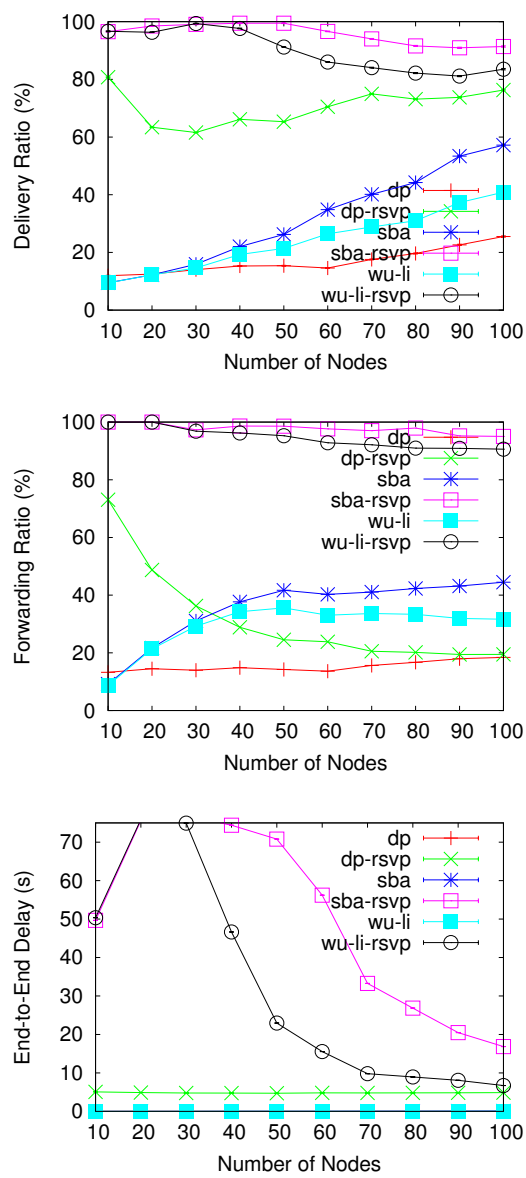

Figure 2. Comparison of Non-Reliable vs. Non-Reliable Extended (R.S.V.P-enabled)

proposed by Peng and Lu [11] (namely, SBA) is that a node does not need to rebroadcast a message that already has been received by neighboring nodes. Whenever a node receives a message, before forwarding, it identifies based on local neighborhood information which nodes should have received the message. If it believes that there are still any other neighbors which have not received the message, it will schedule a re-transmission. Nodes with more neighbors will have a higher priority and will rebroadcast earlier, thus raising the chances of a single transmission reaching a greater number of nodes.

Once again we can see, in Figure 2, how delivery ratio increases when using R.S.V.P. SBA, however, naturally has a higher coverage than Wu and Li's, and consequently involves a larger number of nodes. It is interesting to note that node failure does not have such an effect when using R.S.V.P. In the original SBA, in a fail-free scenario, rates reach $75 \%$ but drop to $57 \%$ with $50 \%$ node failures. The extended SBA's forwarding ratio also peaked at $100 \%$, and the end-to-end delay increased.

Dominant Pruning (DP): The dominant pruning algorithm (namely, DP) [7] is a deterministic neighborhood designating broadcasting protocol that uses 2-hop neighborhood information to reduce redundant transmissions. In DP, whenever a node receives a message, it selects the smallest number of forwarding nodes that can cover all nodes in a 2-hop distance. Although there exist newer algorithms that extend DP, such as [8] where simulation results show that neighborhood information is more effectively used (lower number of gateway nodes) and even more redundant messages are eliminated, they unfortunately seem to produce results which have lower delivery rates. Once again we prefer the original protocol with higher coverage for this work.

In Figure 2 we can see the performance of DP in its original and R.S.V.P-enabled version. DP is the 
protocol with the lowest delivery ratios of all. In the original version delivery ratios reach the maximum of $68 \%$ when in a fail-free scenario, but lower to only $25 \%$ when in a $50 \%$ scenario. In the extended version, however, coverage is always between $63 \%-85 \%$. Unlike previous protocols, neither the forwarding ratio nor the end-to-end delay is that much impacted, but this is mostly due to DP being a neighborhood-designating protocol.

\section{Reliables $v s$. Non-Reliables extended with R.S.V.P}

The biggest challenge behind broadcasting in MANETs still lies in finding the balance between redundancy and reliability. On one hand, many retransmissions can result in more nodes being reached, but so will the chances of collisions and possibly transmission delays rise as well. On the other hand, not enough re-transmissions potentially risks not reaching all nodes. In Figure 3 we show results containing the performance data of both the reliable and the R.S.V.Penabled protocols, which allows us to list a few lessons learned:

- Both RB's flooding approach, as well as EraMobile's gossiping approach maintain the highest delivery ratios of all protocols, but this reliability has its cost: all nodes that receive a message have to act as gateways, as demonstrated in the forwarding ratio results; furthermore, end-to-end delays are unacceptably high.

- All self-pruning protocols extended with R.S.V.P (SBA and $\mathrm{Wu}$ and $\mathrm{Li}$ ) had great improvements regarding reliability, but forwarding ratio jumped to $90 \%$ $100 \%$ range. End-to-end delay also rose considerably, although much less than the RB and EraMobile.

- Most deterministic algorithms rely on correctly updated neighborhood knowledge in order to calculate forward status. But in a fail-prone scenario this information may be misleading. This seems to affect much more neighborhood designating protocols than self-pruning ones as performance results of DCB and DP indicate. However, both of these have much lower forwarding ratios and end-to-end delays.

- Clearly additional mechanisms to properly identify message reception is recommended to determine if all 1-hop and 2-hop neighbors received a message. Nodes must not just assume correct reception. While simply overhearing a retransmission by a neighboring node is one possible solution (as in DCB), depending on reliability requirements of the MANET, it might not be enough.

- For reliability to be ensured, redundancy is a must. Simulations result corroborate this. Efficiency, albeit important, must not be the primary focus of a broadcasting protocol.

\section{Conclusion}

In this paper we proposed a simple reliability metric mechanism called R.S.V.P by which any node is capable of identifying more reliable links prior to transmission. It is a distributed solution that extends deterministic broadcasting algorithms to help enhance a nodes' capability of dealing with omission faults in a scalable scenario. It is interesting to note that when the protocols relied on the R.S.V.P mechanism, the reliability ratios were significantly raised, at the expenses of message re-transmissions and a higher end-to-end delay. Nonetheless, they exhibit end-to-end delays which are still reasonable when compared against the end-to-end results of reliable broadcasting protocols. Based on the simulations results here presented, we can conclude that deterministic broadcasting protocols will greatly benefit from the use of this mechanism since correct message reception by neighboring nodes can be verified, instead of just assumed. A performance comparison with existing reliable broadcasting protocols indicate how efficient R.S.V.P is. As future work we plan on researching how end-to-end delay can be kept at a minimal, thus allowing the usage of R.S.V.P in applications that have timing constraints. We also plan on further improving our failure model to reflect an even more realistic environment.

\section{References}

[1] S. Alagar, S. Venkatesan, and J. Cleveland. Reliable broadcast in mobile wireless networks. In Proc. of Military Communications Conference (MILCOM'95), pages 236-240, November 1995.

[2] M. Ali, U. Saif, A. Dunkels, T. Voigt, K. Romer, K. Langendoen, J. Polastre, and Z. A. Uzmi. Medium access control issues in sensor networks. SIGCOMM Comput. Commun. Rev., 36(2):33-36, 2006.

[3] C. Basile, M. Killijian, and D. Powell. A survey of dependability issues in mobile wireless networks. Technical report, LAAS CNRS Toulouse, France, February 2003.

[4] F. Dai and J. Wu. Distributed dominant pruning in ad hoc networks. In Proceedings of the IEEE 2003 International Conference on Communications (ICC 2003), volume 1, pages 353-357, 2003.

[5] F. Dai and J. Wu. Performance analysis of broadcast protocols in ad hoc networks based on self-prunning. IEEE Transactions on Parallel and Distributed Systems, 15(11):1027-1040, 2004. 
(a) $0 \%$
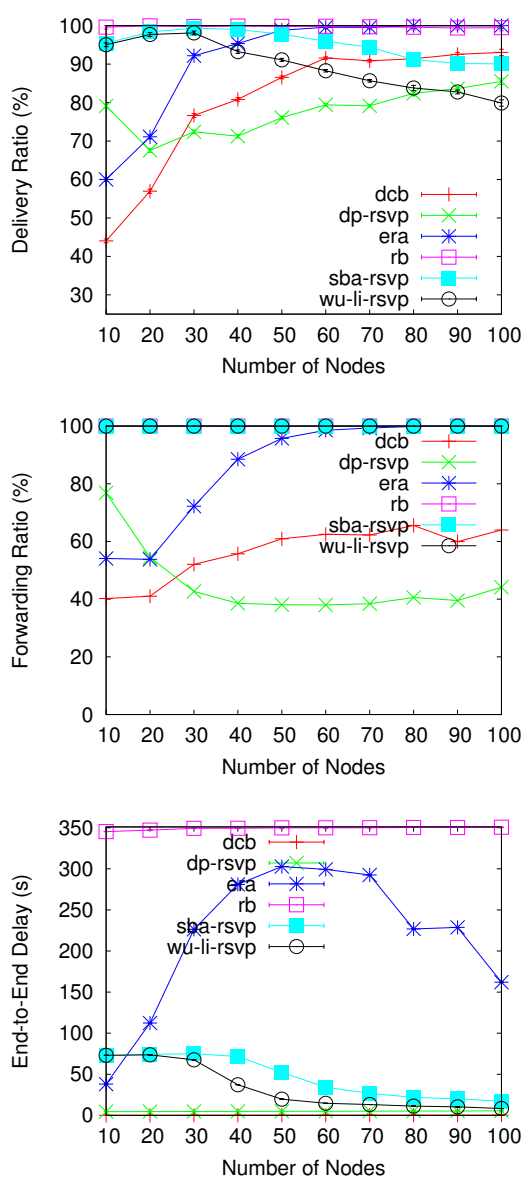

(b) $25 \%$
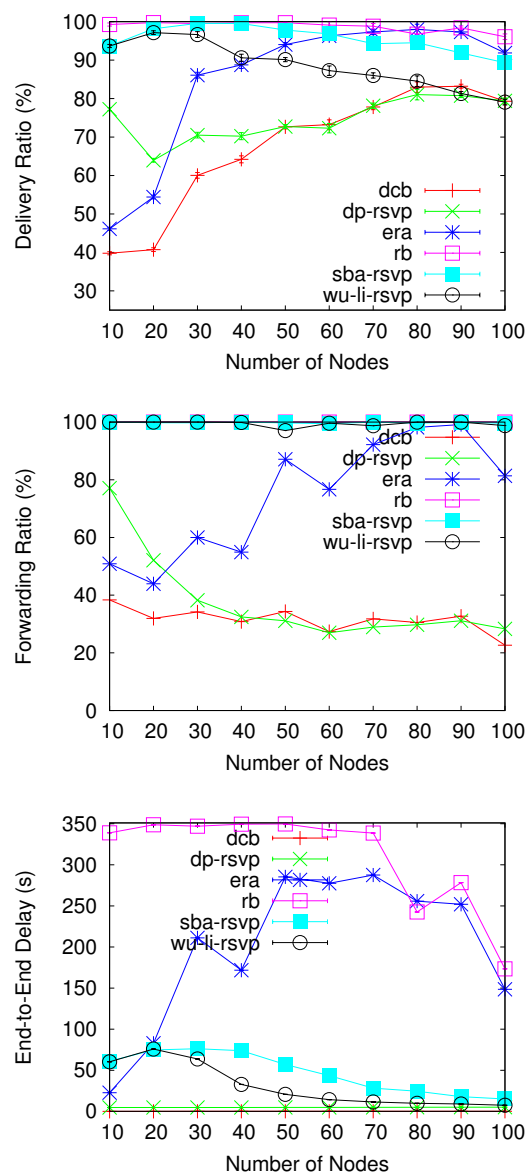

(c) $50 \%$
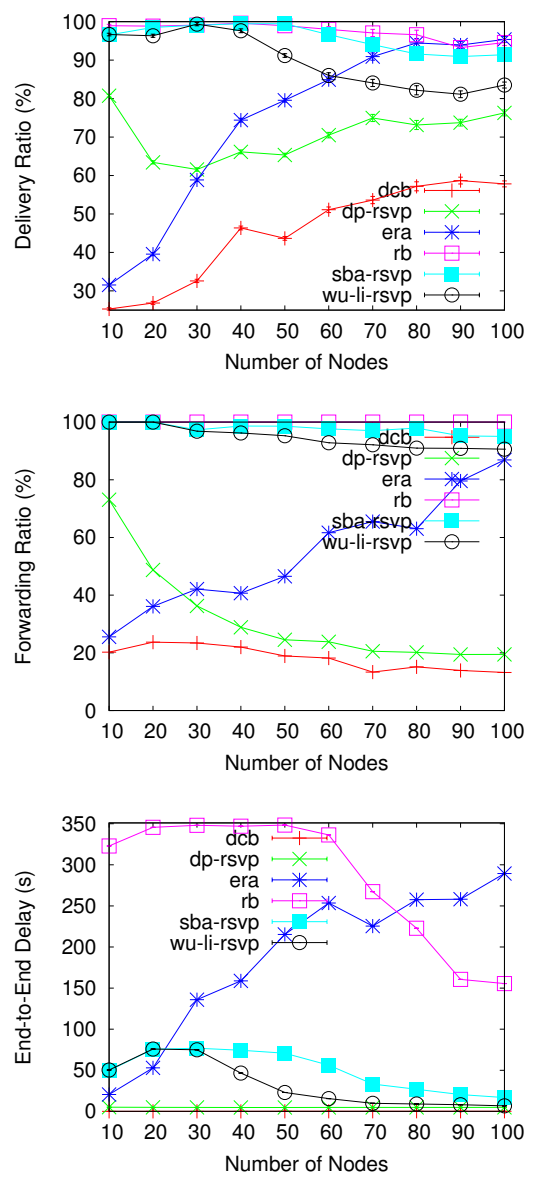

Figure 3. Comparison of Reliable vs. Non-Reliable Extended (R.S.V.P-enabled)

[6] D. Kotz, C. Newport, R. S. Gray, J. Liu, Y. Yuan, and C. Elliott. Experimental evaluation of wireless simulation assumptions. In Proc. of the 7th ACM Int. Symp. on Modeling, Snalysis and Simulation of Wireless and Mobile Systems (MSWiM '04), pages 78-82, New York, NY, USA, 2004. ACM Press.

[7] H. Lim and C. Kim. Flooding in wireless ad hoc networks. Computer Comm., 24(3-4):353-363, 2001.

[8] W. Lou and J. Wu. On reducing broadcast redundancy in ad hoc wireless networks. IEEE Trans. on Mobile Computing, 1(2):111-123, 2002.

[9] W. Lou and J. Wu. Toward broadcast reliability in mobile ad hoc networks with double coverage. IEEE Trans. on Mobile Computing, 6(2):148-163, 2007.

[10] O. Özkasap, Z. Genç, and E. Atsan. Epidemic-based approaches for reliable multicast in mobile ad hoc networks. SIGOPS Oper. Syst. Rev., 40(3):73-79, 2006.

[11] W. Peng and X.-C. Lu. On the reduction of broadcast redundancy in mobile ad hoc networks. In Proc. 1st ACM international symp. on Mobile ad hoc networking
\& computing (Mobihoc), pages 129-130, Piscataway, NJ, USA, 2000. IEEE Press.

[12] E. W. Vollset and P. D. Ezhilchelvan. Design and performance-study of crash-tolerant protocols for broadcasting and reaching consensus in manets. In 24th IEEE Symposium on Reliable Distributed Systems (SRDS 2005), pages 166-175, 2005.

[13] B. Williams and T. Camp. Comparison of broadcasting techniques for mobile ad hoc networks. In Proc. of the 3rd ACM Int. Symp. on Mob. Ad Hoc Networking \& Computing, pages 194-205. ACM Press, 2002.

[14] J. Wu and F. Dai. A generic distributed broadcast scheme in ad hoc wireless networks. IEEE Trans. Computers, 53(10):1343-1354, 2004.

[15] J. Wu and H. Li. On calculating connected dominating set for efficient routing in ad hoc wireless networks. In DIALM '99: Proc. of the 3rd Int. Workshop on Discrete algorithms and Methods for Mobile Computing and Comm., pages 7-14, New York, NY, USA, 1999. ACM Press. 